\title{
Early bolometric luminosity of SN 2013fs without photometry
}

(C) 2020

N. N. Chugai

Institute of Astronomy of Russian Academy of Sciences, Moscow

Keywords: stars - supernovae - SN 2013fs

* email <nchugai@inasan.ru> 


\begin{abstract}
The novel method is proposed for the reconstruction of the early bolometric light curve for supernovae IIP based on a set of spectra of $\mathrm{H} \alpha$ emission during the first day after the shock breakout. The method exploites an effect of the radiative acceleration of the preshock circumstellar gas that is manifested in the broad $\mathrm{H} \alpha$ wings. The efficiency of the method is demonstrated in the case of SN 2013fs with spectra taken between 6 and 10 hours after the shock breakout. The exceptional feature of this method is that it does not require the photometry, the distance, and the extinction.
\end{abstract}

\title{
1. Introduction
}

A type IIP supernova (SN IIP) is an outcome of the explosion of a red supergiant (RSG) caused by the core-collapse. Following the explosion, ejecta expands in the environment created by the presupernova mass loss. The RSG wind of pre-SN IIP has a moderate density parameter $\dot{M} / u \sim 10^{14}-10^{15} \mathrm{~g} \mathrm{~cm}^{-1}$ indicated by the radio and X-ray emission (Chevalier et al. 2006). The optical emission lines, e.g., $\mathrm{H} \alpha$ from the wind of that density are generally expected to be weak for the detection. Yet in several SNe IIP the spectra show during first couple days rather strong narrow emission lines apparently originated from a confined ionized dense circumstellar (CS) shell (Quimby et al. 2007; Groh et al. 2014; Khazov et al. 2016; Yaron et al. 2017). The most interesting case is SN 2013fs with spectra starting from $6 \mathrm{~h}$ after the shock breakout (Yaron et al. 2017). The analysis of these spectra led authors to conclude that the early CS emission lines originate from the confined shell $\left(r<10^{15} \mathrm{~cm}\right)$ with the mass of $($ several $) \times 10^{-3} M_{\odot}$, the expansion velocity of $50-100 \mathrm{~km} \mathrm{~s}^{-1}$, and the Thomson optical depth of $\tau_{T} \sim 1-2$ (Yaron et al. 2017).

The line profiles of early spectra of SN 2013fs show narrow core and extended wings very much similar to emission lines of early SN 1998S, in which case they have been attributed to the emission from the dense CS shell with the optical depth to the Thomson 
scattering of $\tau_{T} \sim 3-4$ (Chugai 2001). However this interpretation fails for SN 2013fs because broad wings observed in $\mathrm{H} \alpha$ are more intense compared to the model of the Thomson scattering; moreover the blue wing is stronger than the red one unlike the model (Yaron et al. 2017). The problem has been resolved in the model that includes the acceleration of the preshock CS gas by the SN radiation (Chugai 2020a); for the H $\alpha$ at $10.3 \mathrm{~h}$ the recovered preshock $\mathrm{CS}$ velocity is $3000 \mathrm{kms}^{-1}$. The broad $\mathrm{H} \alpha$ wings in early spectra of SN 2013fs are essentially dominated by the emission from the accelerated CS gas and not by the Thomson scattering. Remarkably, in SN 1998S the effect of the radiative acceleration of the circumstellar gas upto $1000 \mathrm{~km} \mathrm{~s}^{-1}$ is overwehlmed by the Thomson scattering (Chugai 2001). Dessart et al. (2017) explored numerically effects of dense CS shell around SN IIP using radiation hydrodynamics and non-LTE radiation transfer. The models predict significant acceleration of the CS gas upto $>5000 \mathrm{~km} \mathrm{~s}^{-1}$ during first couple days. However the reported synthetic spectra do not indicate, in which cases line wings are dominated by either Thomson scattering or the bulk velocity of the accelerated CS gas.

The proposed explanation of the the early $\mathrm{H} \alpha$ spectrum of SN 2013fs prompts a possibility that the preshock velocity inferred from the early $\mathrm{H} \alpha$ can be used to probe the early bolometric luminosity of SN 2013fs that otherwise is poorly determined. Here I present the method for the reconstruction of the early bolometric light curve of SN 2013fs based on preshock velocity extracted from the $\mathrm{H} \alpha$ emission line. To this end we first determine velocities of the preshock CS gas from the $\mathrm{H} \alpha$ in Keck-I spectra between $6 \mathrm{~h}$ and $10 \mathrm{~h}$ after the shock breakout. The found velocities are used then to recover the bolometric luminosity of SN 2013fs during first 10 hours after the shock breakout. The study exploits spectra of SN 2013fs retrieved from the database WISeREP (Yaron \& GalYam 2012) (https://wiserep.weizmann.ac.il).

\section{Model}


The shock breakout of an exploding star with an extended envelope is accompanied with the sweeping of the outmost atmosphere into the dense shell (Grasberg et al. 1971). For the exploding RSG the mass of the thin dense shell is $10^{-4}-10^{-3} M_{\odot}$ (Chevalier 1981). The thin shell decelerates in the CS gas that results in the formation of the forward and reverse shock with the thin dense shell inbetween (Chevalier 1982a; Nadyozhin 1985). The reverse shock is generally radiative, so the shocked cold ejecta are accumulated in the thin dense shell that we dub the cold dense shell (CDS) since its kinetic temperature is lower than the temperature of both shocks.

The size and density of the dense CS shell of SN 2013fs can be recovered from spectral data in the following way. On day 2.42 the spectrum shows the broad He II $4686 \AA$ emission (Bullivant et al. 2018) that is attributed to the fragmented CDS with the expansion velocity of $v_{c d s}=16600 \mathrm{~km} \mathrm{~s}^{-1}$ (Chugai 2020b). Narrow $\mathrm{H} \alpha$ associated with the CS shell disappeares between epochs of Keck-II spectra on day 2.1 and 5.1 (Yaron et al. 2017). This means that at about $t \sim 3 \mathrm{~d}$ the CS shell is overtaken by the CDS, which gives us the extension of the CS shell $R_{c d s} \sim v_{c d s} t \sim 5 \times 10^{14} \mathrm{~cm}$. The $\mathrm{H} \alpha$ modelling in the spectrum on $10.3 \mathrm{~h}$ implies the Thomson optical depth of the CS shell $\tau_{T} \sim 2$ (Chugai 2020a). The average electron number density in the CS shell is thus $n_{e}=\tau_{T} /\left(R_{c s} \sigma_{T}\right) \sim 6 \times 10^{9} \mathrm{~cm}^{-3}$ and the density $\rho_{0}=1.2 \times 10^{-14} \mathrm{~g} \mathrm{~cm}^{-3}$ assuming hydrogen abundance $X=0.7$. Below we adopt homogeneous CS shell with the density $\rho_{0}=1.35 \times 10^{-14} \mathrm{~g} \mathrm{~cm}^{-3}$ that is by a factor of three higher compared to the model that is aimed at the minimization of the explosion energy of SN 2013fs (Chugai 2020b).

One can apply the hydrodynamics in the thin shell approximation (cf. Chugai 2020b) to find the CDS dynamics that matches the expansion velocity on day 2.42. The rate of the CDS decceleration is determined by the CS density and the SN ejecta density distribution in outer layers; the latter generally follows the power law $\rho(v)=\rho_{1}\left(t_{1} / v\right)^{3}\left(v_{1} / v\right)^{q}$. We adopt $q=7.6$ in line with the hydrodynamic model of the normal type IIP SN 2008in (Utrobin \& Chugai 2013). For the reference values $t_{1}=1 \mathrm{~d}, v_{1}=10^{4} \mathrm{~km} \mathrm{~s}^{-1}$ the CDS expansion satisfies the velocity constrain on day 2.42 (Fig. 1) for $\rho_{1}\left(t_{1}, v_{1}\right)=3.44 \times$ 
$10^{-10} \mathrm{~g} \mathrm{~cm}^{-3}$.

At the initial phase of $\sim 1$ day the photosphere of SN 2013fs coincides with the CDS. Indeed, the momentum flux conservation suggests that the CDS density is $\sim \rho_{0}\left(v_{c d s} / c_{s}\right)^{2} \sim$ $6 \times 10^{-9} \mathrm{~g} \mathrm{~cm}^{-3}$ (Grasberg et al. 1971), where $c_{s} \approx 37 \mathrm{~km} \mathrm{~s}^{-1}$ is the isothermal sound speed for the CDS temperature of $T \sim\left(L / 4 \pi r^{2} \sigma\right)^{0.25} \sim 3.5 \times 10^{4} L_{43}^{0.25} / r_{14}^{0.5} \mathrm{~K}$. For these conditions the Rosseland opacity $k_{R} \sim 2$ (Badnell et al. 2005). With the CDS mass of $\sim 3 \times 10^{-4} M_{\odot}$ and the CDS radius at $t=10 \mathrm{~h}$ of $R_{c d s} \sim 10^{14} \mathrm{~cm}$ (Fig. 1 ) the CDS optical depth turns out to be $\tau=k_{R} M_{c d s} /\left(4 \pi R_{c d s}^{2}\right) \sim 10$, so indeed the CDS at the considered phase is opaque and the photosphere resides at the CDS.

The overall picture for the $\mathrm{H} \alpha$ formation at the considered stage thus can be described as a photosphere $(\mathrm{CDS})$ of the radius $R_{c d s}$ enclosed by the hot forward shock of the radius $R_{s} \approx \xi R_{c d s}$ that is embedded into the ionized confined CS shell $R_{s}<r<R_{c s}$. Noteworthy that the $\mathrm{H} \alpha$ model is not sensitive to the parameter $\xi$. We adopt $\xi=1.2$ that corresponds to the self-similar solution for $q=7$ and the uniform CS medium (Chevalier 1982). The forward shock layer is assumed to be uniform with the density of $4 \rho_{0}$. The high number density in the forward shock $\left(n \sim 3 \times 10^{10} \mathrm{~cm}^{-3}\right)$ implies the fast electronproton thermal equilibration compared to the expansion time, so the electron temperature is $T_{e}=1.6 \times 10^{9} v_{s, 4}^{2}$, where $v_{s, 4}=v_{s} / 10^{4} \mathrm{~km} \mathrm{~s}^{-1}$. The radiative cooling time is significantly longer than the expansion time, however the Compton cooling time $t_{C}=1.2 \times 10^{4} r_{14} L_{43}^{-1}$ s can be comparable to the expansion time; the postshock electrons can therefore cool by a factor of $\sim 2$. We therefore adopt $T_{e}=10^{9} \mathrm{~K}$ through the postshock layer. Noteworthy that the $\mathrm{H} \alpha$ profile is not sensitive to the variation of the electron temperature even by a factor of ten.

The powerful early SN radiation results in the significant acceleration of the CS gas with the velocity being maximal just before the shock and decreasing outwards. We describe the velocity distribution of the CS gas at the fixed moment by the expression

$$
v(r)=\left(v_{p s}-v_{c s}\right)\left(\frac{R_{c s}-r}{R_{c s}-R_{s}}\right)^{s}+v_{c s},
$$


where $v_{p s}$ is the preshock CS velocity at $r=R_{s}$ and $v_{c s}$ is the velocity of the undisturbed CS gas at $r=R_{c s}$. The value of power index is $s \approx 1.6$ for optimal $\mathrm{H} \alpha$ models.

\section{Results}

\subsection{CS gas velocity}

The radiation transfer of $\mathrm{H} \alpha$ photons in the CS shell is treated using the Monte Carlo technique. The $\mathrm{H} \alpha$ emission is dominated by the recombination mechanism, so the emissivity in the uniform CS envelope is assumed to be constant along the radius. The model radiation transfer takes into account resonanant scattering in $\mathrm{H} \alpha$ in the Sobolev approximation. However the previous modelling of the $\mathrm{H} \alpha$ at $10.3 \mathrm{~h}$ (Chugai 2020a) indicates that the Sobolev optical depth in $\mathrm{H} \alpha$ should be negligibly small. This situation reflects strong depopulation of the hydrogen second level due to the high photoionization rate by the SN radiation.

The angle-averaged frequency redistribution function (Mihalas 1978) is applied for the photon scattering on thermal electrons. The $\mathrm{H} \alpha$ profile is not sensitive to the exact value of the CS electron temperature since the broadenning is dominanted by the high velocities of the radiatively accelerated CS gas. Nevertheless the modelling takes into account the evolution of the electron temperature. At the first iteration we use the value $T_{e}=4 \times 10^{4} \mathrm{~K}$ for all the considered epochs. With this temperature we recover CS velocity from $\mathrm{H} \alpha$, the bolometric luminosity and the effective temperature. This temperature is adopted as the electron temperature and these values of $T_{e}$ are used for the final $\mathrm{H} \alpha$ models. The radiation transfer includes a diffuse reflection of photons from the photosphere. However this effect for $\mathrm{H} \alpha$ photons is equivalent to zero albedo because reflected photons scatter in the far blue wing due to the high photosphere velocity of $\gtrsim 26000 \mathrm{~km} \mathrm{~s}^{-1}$ at the considered epoch (Fig. 1). 
The optimal $\mathrm{H} \alpha$ models are overplotted on the observed low resolution $\left(160 \mathrm{~km} \mathrm{~s}^{-1}\right)$ spectra between $6 \mathrm{~h}$ and $10 \mathrm{~h}$ (Fig. 2) with parameters given in the Table 1 . The table columns contain the time after the shock breakout, the CDS radius, the electron temperature of the CS gas, the preshock Thomson optical depth, and the preshock velocity inferred from the $\mathrm{H} \alpha$ fit. The bottom line includes the result obtained earlier for the high resolution spectrum at $10.3 \mathrm{~h}$ (Chugai 2020a). The uncertainty of the inferred velocity is in the range of $20 \%$. The similar uncertainty refers to the Thomson optical depth recovered from $\mathrm{H} \alpha$. The primary indicator of the Thomson optical depth is the profile skewing towards blue being apparent in all the profiles of Fig. 2. However, $\tau_{T}$ in Table 1 are taken from the interaction model for consistency; these values are used for the $\mathrm{H} \alpha$ model.

The crucial role of the radiative acceleration of the CS gas for the $\mathrm{H} \alpha$ profile is emphasised by the $\mathrm{H} \alpha$ computed without radiative acceleration effect, and using the constant velocity of $100 \mathrm{~km} \mathrm{~s}^{-1}$ for the CS shell (Fig. 2). It is clear that the Thomson scattering exclusively cannot account for the observed H $\alpha$. This differs SN 2013fs from SN 1998S where the Thomson scattering dominates over the effect of the moderate radiative acceleration of $1000 \mathrm{~km} \mathrm{~s}^{-1}$ (Chugai 2001).

\subsection{Early bolometric luminosity}

Preshock CS velocity $v_{p s}$ is a direct indicator of the radiation energy $E_{r}$ emitted by the supernova between the shock breakout and the observation epoch. The radiative force is dominated by the Thomson scattering for conditions in the CS shell (cf. Chugai et al. 2002). Neglecting the CS gas displacement the solution of the equation of motion of the CS gas in the field of SN radiation results in the preshock velocity

$$
v_{p s}=\frac{k_{T} E_{r}}{4 \pi R_{s}^{2} c}
$$

where $k_{T}=0.34 \mathrm{~cm}^{2} \mathrm{~g}^{-1}$ is the coefficient of the Thomson scattering, $c$ is the speed of light. 
The inferred $v_{p s}$ values (Table 1) with the equation (2) permit us to recover $E_{r}$ for the explored moments. We apply two different descriptions of the initial stage of the luminosity decline: the exponential law $L=L_{0} \exp \left(-t / t_{0}\right)$ and the power law $L=L_{0} /\left[1+\left(t / t_{0}\right)^{p}\right.$. In each case parameters are determined by the $\chi^{2}$ minimization. For the exponential law $t_{0}=0.12 \mathrm{~d}$ and $L_{0}=7.23 \times 10^{44} \mathrm{erg} \mathrm{s}^{-1}$, whereas for the power law $t_{0}=0.12 \mathrm{~d}$, $L_{0}=5.8 \times 10^{44} \mathrm{erg} \mathrm{s}^{-1}$, and $p=2.6$ (Fig. 31). Both descriptions for the luminosity coincide within $10 \%$, while the energy radiated during initial $0.5 \mathrm{~d}$ in both cases is $7.4 \times 10^{48} \mathrm{erg}$. The relative error of $E_{r}$ is the same as that of the velocity, i.e. $20 \%$.

The recovered bolometric luminosity is compared (Fig. 3, panel b) to the reported bolometric light curves obtained from the multiband photometry in two ways (Yaron et al. 2017). The first approach suggests the reconstruction of the spectral energy distribution (SED), while the second method is based on the recovered blackbody temperature, photo-

spheric radius, and the blackbody luminosity $L=4 \pi R^{2} \sigma T^{4}$. These two reported versions differ by a factor of 100 (Fig. 3, panel b) at the first observational epoch that emphasises difficulties of the early light curve reconstruction from the photometric data. Amazingly, our light curve is consistent with the blackbody version of the reported light curve despite of radically different methods.

It is noteworthy that the early bolometric light curve of SN 2013fs recovered from the radiative acceleration effect in $\mathrm{H} \alpha$ does not requires the photometry, the distance, and the extinction. The point is that this method exploits only measurements of the velocities based on spectra expressed in relative fluxes.

\section{Conclusion}

The paper has been aimed at the reconstruction of the early bolometric light curve of SN 2013fs exploiting effect of the radiative acceleration of the CS gas being apparent in the $\mathrm{H} \alpha$ emission. The proposed method turns out to be a success in the case of SN $2013 \mathrm{fs}$ thanks to the unique set of Keck-I spectra between $6-10.3 \mathrm{~h}$ after the shock breakout. The 
attractive feature of the novel method is that the early bolometric light curve of SN $2013 \mathrm{fs}$ is recovered disregarding the photometry, distance, and extinction.

Some uncertainty might stem from the choice of a function that approximates the luminosity decline after the shock breackout. In fact this arbitrariness does not affect the result. Two different choices, exponential and power law, result in the bolometric light curves that agree with each other within 10\%; moreover the total radiation emitted during 0.5 day after the shock breakout in both cases is the same. Suprisingly, that the recovered bolometric luminosity is close to the luminosity estimated using blackbody approximation for the moments 0.16, 0.36, and 0.55d ((Yaron et al. 2017). The agreement between results obtained by two completely different approaches indicates that both methods catch behavior of the real luminosity of SN 2013fs at the initial stage.

Yet unlike the case of SN 2013fs with the known distance 47-51 Mpc (NED) and small extinction (Yaron et al. 2017), for nearby SNe IIP with less certain distance and extinction sytematic errors in the bolometric luminosity recovered from the photometry can be large. All three factors of the uncertainty (distance, extinction, and photometric incompleteness) are lacking in the new method. It should be emphasised potential significance of the new method for the reconstruction of the early bolometric luminosity of a future SN IIP in our Galaxy, since in that case the distance and the extinction will be known likely with large uncertainties. Obviously, a successfull application of the proposed method requires the observation of a set of spectra during the first day after the explosion. 


\section{References}

Badnell N.R., Bautista M.A., Butler K., et al., Mon. Not. R. Astron. Soc. 360, 458 (2005)

Chevalier R.A., Astrophys. J. 259, 302 (1982)

Chevalier R.A., Fransson C., Nymark T.K., Astrophys. J. 641, 1029 (2006)

Chevalier R.A., Fundamentals of Cosmic Physics. 7, 1 (1981)

Chugai N.N., Astron. Lett. 46, 319 (2020a)

Chugai N.N., Mon. Not. R. Astron. Soc. 494, L86 (2020b)

Chugai N.N., Blinnikov S.I., Fassia A. et al. Mon. Not. R. Astron. Soc. 330, 473 (2002)

Chugai N.N., Mon. Not. R. Astron. Soc. 326, 1448 (2001)

Dessart L., Hillier D.J., Audit E., Astron. Astrophys. 605, A83 (2017)

Grasberg E.K., Imshennik V.S., Nadyozhin D.K., Astrophys. Space Sci. 10, 3 (1971)

Groh J.H., Astron. Astrophys. 572, L11 (2014)

Khazov D., Yaron O., Gal-Yam A. et al., Astrophys. J. 818, 3 (2016)

Quimby R.M, Wheeler J.C., Höflich P. et al., Astrophys. J. 666, 1093 (2007)

Shivvers I., Groh J.H., Mauerhan J. C. et al., Astrophys. J. 806, 213 (2015)

Utrobin V.P, Chugai N.N., Astron. Astrophys. 555, A145 (2013)

Yaron O., Perley D.A., Gal-Yam A. et al., Nature Physics 13, 510 (2017)

Yaron O., Gal-Yam A., Publ. Astron. Soc. Pacific 124, 668 (2012) 
Table 1. Parametrs of $\mathrm{H} \alpha$ model

\begin{tabular}{l|c|c|c|c}
\hline Day & $R_{c d s}\left(10^{14} \mathrm{~cm}\right)$ & $T_{e}$ & $\tau_{T}$ & $v_{p s}\left(\mathrm{~km} \mathrm{~s}^{-1}\right)$ \\
\hline 0.258 & 0.74 & $60000 \pm 3000$ & 1.9 & $7500 \pm 1500$ \\
0.30 & 0.85 & $50000 \pm 2500$ & 1.9 & $6000 \pm 1200$ \\
0.371 & 1.05 & $40000 \pm 2000$ & 1.8 & $4000 \pm 800$ \\
0.421 & 1.18 & $35000 \pm 1800$ & 1.7 & $3500 \pm 700$ \\
0.423 & 1.19 & $35000 \pm 1800$ & 1.7 & $3000 \pm 600$ \\
\hline
\end{tabular}




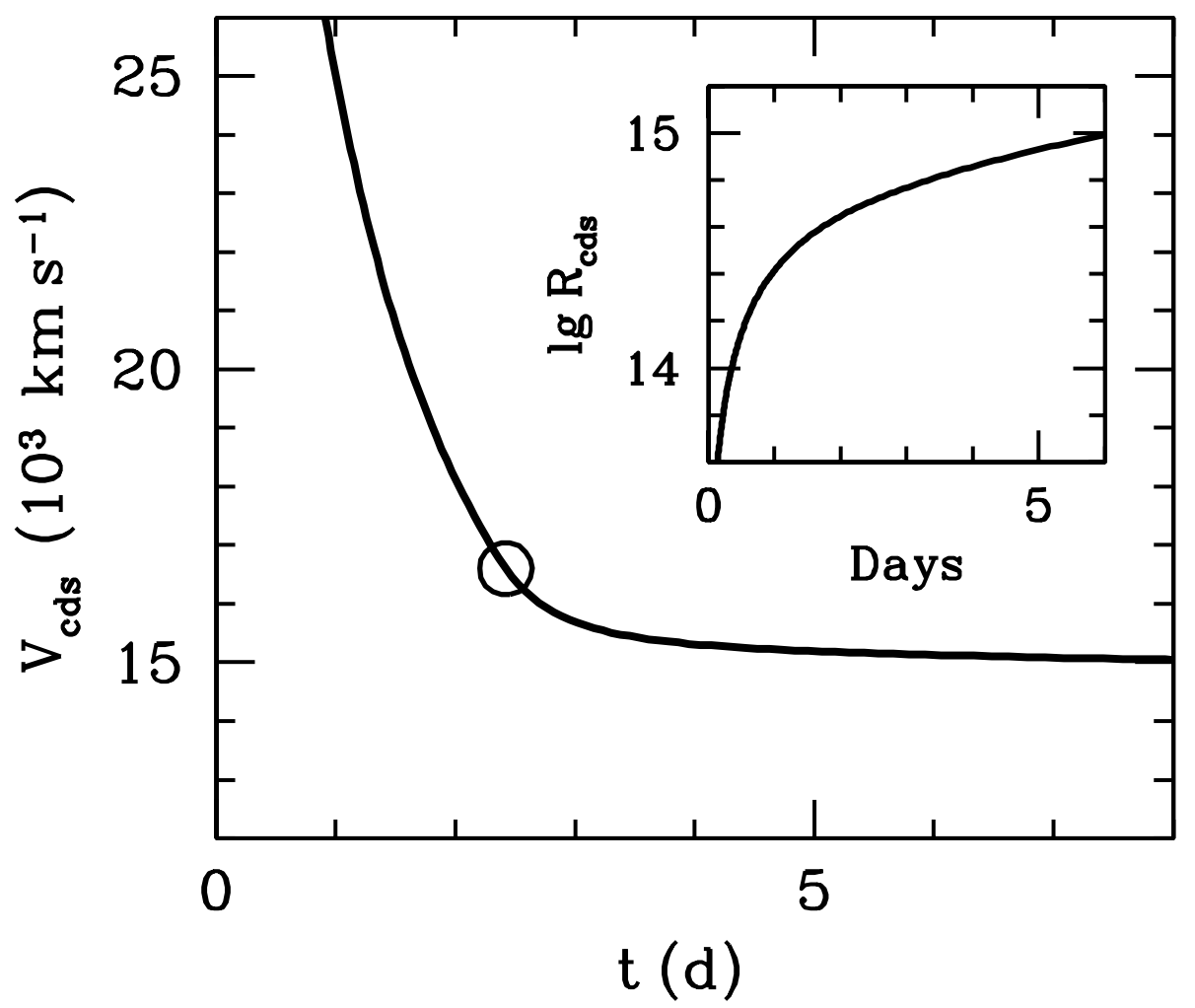

Figure 1: The model velocity of the cold dense shell fits the obeservational estimate (circle) from HeII $4686 \AA$ line on day 2.4. Insert shows the evolution of the model CDS radius. 

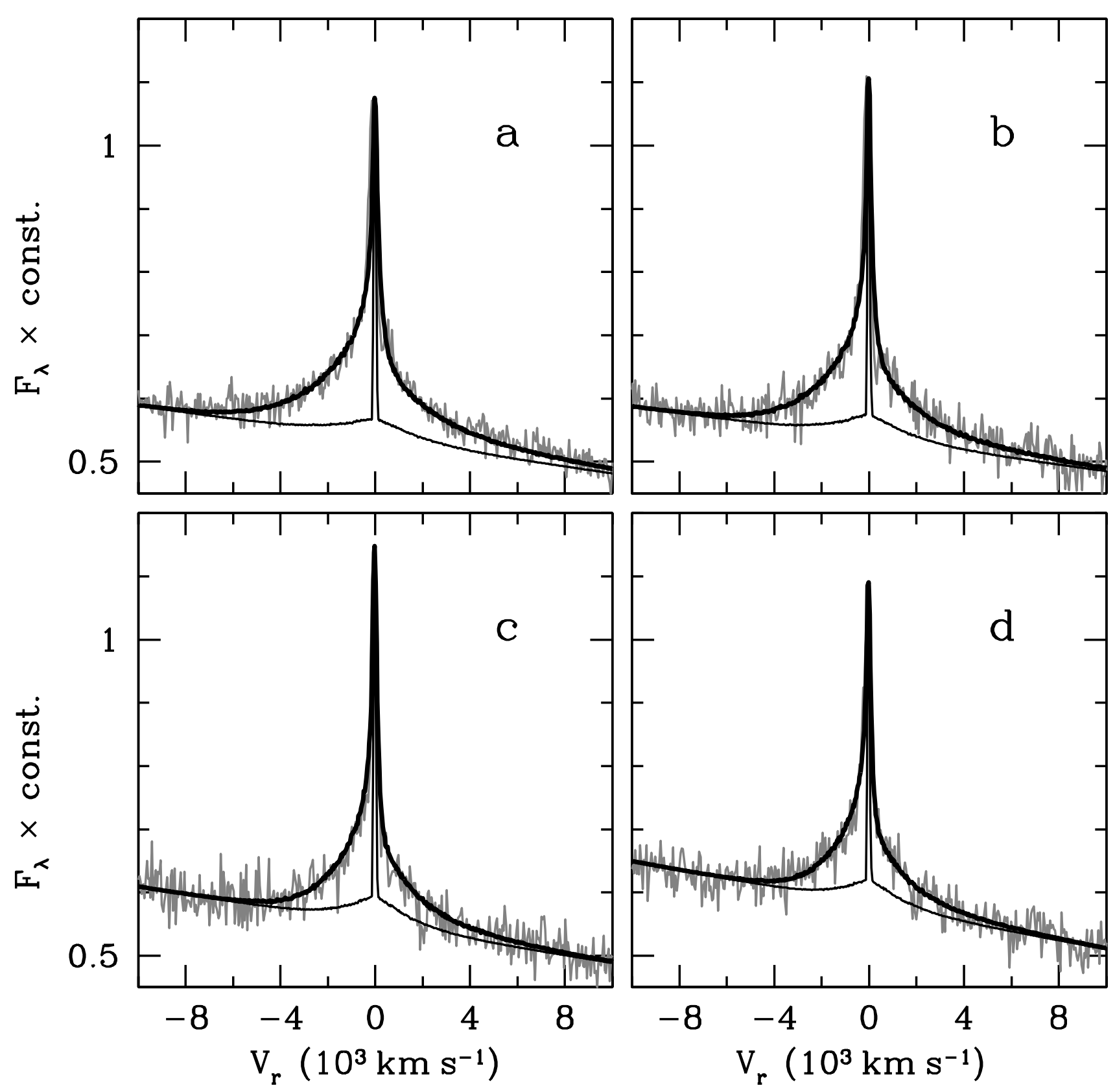

Figure 2: $\mathrm{H} \alpha$ emission in spectra of SN 2013fs. Model $\mathrm{H} \alpha$ (thick line) is overploted on observed spectra (grey) taken at the moments $0.26 \mathrm{~d}$ (panel a), $0.3 \mathrm{~d}(\mathbf{b}), 0.37 \mathrm{~d}$ (c), and $0.42 \mathrm{~d}(\mathbf{d})$ after the shock breakout. Thin black line shows models with the constant CS gas velocity of $100 \mathrm{~km} \mathrm{~s}^{-1}$. 

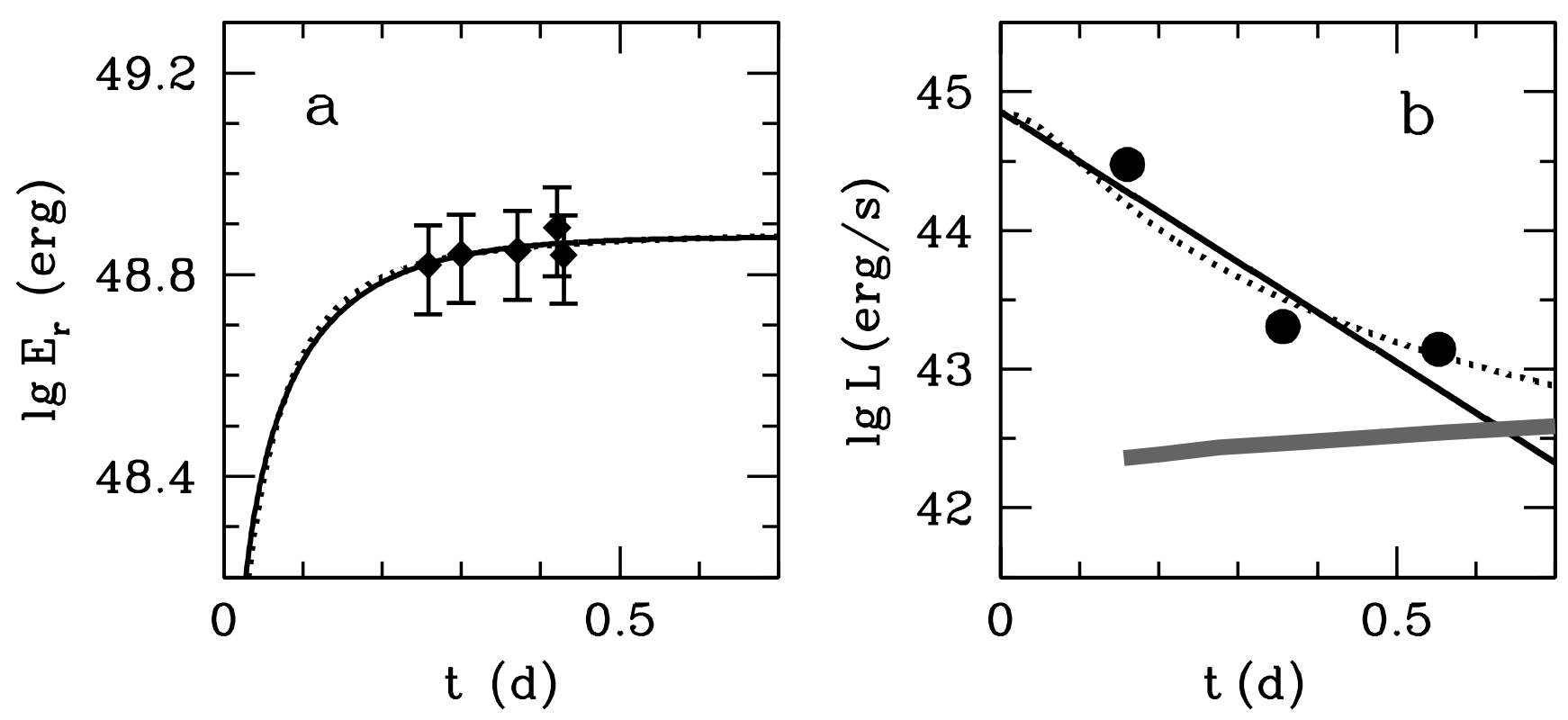

Figure 3: Radiation energy emitted by SN 2013fs (panel a) recovered from the preshock velocities (diamonds) with the overploted luminosity presented by the exponential luminosity decline (solid line) and the power law (dotted). Panel $\mathbf{b}$ shows the recovered luminosity for the exponential law (black line) and power law (dotted line) that fit the radiation energy in panel a. Circles correspond to the reported luminosity (Yaron et al. 2017) recovered from the multiband photometry based on the black body approximation, while the grey line is the luminosity recovered from the multiband photometry based on the SED reconstruction. 